\title{
Protection against salinity stress in black cumin involves karrikin and calcium by improving gas exchange attributes, ascorbate-glutathione cycle and fatty acid compositions
}

\author{
Parisa Sharifi ${ }^{1}\left[\right.$. Siamak Shirani Bidabadi ${ }^{2}$
}

Received: 4 July 2020 / Accepted: 27 October 2020 / Published online: 18 November 2020

C) Springer Nature Switzerland AG 2020

\begin{abstract}
The present study assessed the effect of karrikin (KAR) and calcium $\left(\mathrm{CaCl}_{2}\right)$ to increase salt tolerance in black cumin. Salinity stress induced oxidative stress by enhancing electrolyte leakage (EL), malondialdehyde (MDA) and hydrogen peroxide $\left(\mathrm{H}_{2} \mathrm{O}_{2}\right)$ content while having a negative effect on seed yield, fatty acid content and composition, total chlorophyll content, chlorophyll fluorescence and leaf gas exchange parameters of black cumin. The activity of antioxidant enzymes involved in the ascorbate-glutathione cycle was also affected by salt stress. Black cumin seed trimming with karrikin when followed with calcium foliar application mitigated the harmful effects of salinity by improving chlorophyll content, chlorophyll fluorescence and leaf gas exchange parameters while reducing the $\mathrm{H}_{2} \mathrm{O}_{2}$ generation, MDA accumulation and EL. Calcium application in karrikin pretreated seedlings also reversed salinity-induced changes by enhancing the activities of enzymes in the ascorbate-glutathione cycle, other antioxidants (superoxide dismutase, catalase) and osmoprotectant (proline). The ability of black cumin seedlings to cope with salt stress after pretreatment with KARs when followed by external application of calcium can be attributed to increased levels of polyunsaturated fatty acids and modification of enzymatic and nonenzymatic antioxidants as well as enhanced osmolyte accumulation.
\end{abstract}

Keywords Antioxidant systems $\cdot \mathrm{CaCl}_{2} \cdot$ Karrikin $\cdot$ Osmoprotectant $\cdot$ Oxidative stress $\cdot$ Salinity

\section{Introduction}

Black cumin (Nigella sativa L.) is an important oilseed crop of the Ranunculaceae family, native to the semiarid regions of Mediterranean, which are the highest areas of cultivation in South Europe, Syria, Saudi Arabia, Iran, Pakistan, India and Turkey [1]. It is a rich source of antioxidant, oil and protein and therefore cultivated in many countries for both nutritional and medicinal value [2]. In addition to the fact that the amount of unsaturated fatty acids in black cumin seeds is $69.7-73.5 \%$ and its saturated fatty acid is much lower (24.8-29.2\%), the presence of some important medicinal effects such as antiseptic, antihistamine, antihypertensive, anti-inflammatory, antitumor and insect repellent has also made the plant more valuable $[3,4]$.

Since salinity damages the agricultural production billions of dollars annually, it is a serious constraint for crop yields and sustainable agricultural productivity, especially in arid and semiarid regions [5, 6]. Improper irrigation practices in arid and semiarid regions and the use of saline and alkaline water for irrigation have made salinity stress one of the major challenges facing crop yields [7]. High salinity concentrations have caused about $7 \%$ of the total land area to be destroyed and uncultivated [8, 9]. Salinity may negatively affect the physiological processes of plant crops, one of which is the enhanced oxidative stress

Parisa Sharifi, pa.sharifi@urmia.ac.ir | ${ }^{1}$ Department of Agricultural Extension and Education, Higher Education Center Shahid Bakeri Miyandoab, Urmia University, Urmia 94171-71946, Iran. ${ }^{2}$ Department of Horticulture, College of Agriculture, Isfahan University of Technology, Isfahan 84156-83111, Iran. 
leading to the formation of reactive oxygen species (ROS) $[10,11]$.

Karrikinolide is an active chemical compound of 3-methyl-2H-furo [2, 3-c] pyran-2-one called karrikins (KARs) [10, 12-15]. Karrikins, caused by smoke from the combustion of plant materials, are among the newly discovered groups of plant growth regulators [16]. Recent findings have provided evidence that KARs have a potential role in enhancing abiotic stress tolerance in plants [13, 17-19]. Calcium plays a very positive role in moderating the destructive effects of salinity and reducing the toxicity of $\mathrm{NaCl}$ in plants [20]. Results from previous studies show that calcium increases the activity of antioxidant enzymes, decreases lipid peroxidation and regulates the osmotic pressure of plant cells under salinity stress [21, 22]. When applied $\mathrm{Ca}^{2+}$ is taken up, it consequently modifies plant responses to several abiotic stresses by involving directly or indirectly in defense systems. Calcium-applied alleviation of salinity-induced damage has been expressed in several plants such as Calligonum mongolicum [23], Tomato [24], Cakile maritime [22], Nitraria tangutorum [25].

The defensive role of both $\mathrm{Ca}^{2+}$ and karrikins in oxidative stress has been reported to be connected with the elevated activities of antioxidant enzymes, thereby regulating redox homeostasis under salt stress $[26,27]$. This has led to the hypothesis in our minds that calcium application in karrikin pretreated black cumin may alleviate salinity stress. Therefore, it is very important to follow the responses of black cumin at physiological level to salinity stress and inquire how physiological processes are affected by KARs and calcium applications to alleviate the decrease in photosynthesis attributes, reduce oxidative stress and improve lipids and fatty acid compositions under salt stress. It was hypothesized that KARs and calcium additions in this study can overcome the harmful effects of $\mathrm{NaCl}$ stress on the medicinal plant Nigella sativa.

\section{Materials and methods}

This research was carried out in pots in the research greenhouses of Urmia University, Urmia, Iran, during the year 2018. Healthy and uniform seeds of black cumin (Nigella sativa) purchased from Isfahan Pakan Bazr Co., Iran, were surfacesterilized for $5 \mathrm{~min}$ with $5 \%$ sodium hypochlorite $(\mathrm{NaClO})$ and then washed carefully with double-distilled water. The seeds were primed with karrikin $(10 \mu \mathrm{mol})$ for $16 \mathrm{~h}$ before sowing and then planted in plastic pots $(30 \mathrm{~cm}$ diameter and $50 \mathrm{~cm}$ height) filled with $10 \mathrm{~kg}$ of oven-dried soil (sandy soil). The experimental pots were arranged in a completely randomized design in the greenhouse with average day/ night temperatures of $24 / 18^{\circ} \mathrm{C}$, relative humidity of $70-75 \%$, light intensity of $3700 \mu \mathrm{mol} \mathrm{m}^{-2} \mathrm{~s}^{-1}$ and photoperiod of $18 \mathrm{~h}$ light/ $6 \mathrm{~h}$ dark, according to Khalid [28]. Three weeks after the seeds were sown, seedlings obtained were thinned to three uniform plants per pot. Calcium solution prepared in the form of calcium chloride dihydrate $\left[\mathrm{CaCl}_{2} \cdot 2 \mathrm{H}_{2} \mathrm{O}\right]$ (SigmaAldrich, USA) was employed at different levels $(0,50$ and $100 \mathrm{mmol}$ ) containing $0.1 \%$ tween- 20 as surfactant. Spraying was carried out four times throughout the experiment, including 3, 6, 9 and 12 weeks of sowing date. To ensure a uniform distribution of the $\mathrm{Ca}^{2+}$ solution on all leaves in each plant, a compressor sprayer of $1 \mathrm{~L}$ capacity was employed. Distilled water of equal volume of calcium solutions was used as foliar spray for noncalcium treatment (control). Salinity treatments $(0,50,100$ and $150 \mathrm{mmol} \mathrm{NaCl})$ were started 6 weeks after germination and continued until the end of the experiment which lasted for 15 weeks. Seedlings were irrigated with saline treatments every 7 days. In order to avoid excessive salt accumulation in the pots, all pots were drained once every 28 days by distilled water. Each treatment had three replications (pots), so that three seedlings were planted in each pot. At the end of the tests which lasted for 15 weeks, seeds were harvested at the fruiting stage and weight and seed yield (g plant ${ }^{-1}$ ) were then recorded.

\subsection{Chlorophyll content and Fv/Fm measurement}

Relative leaf chlorophyll content was measured using spectrophotometry (Model Apel PD-303S, Apel Co. Ltd, Japan) on the fully developed upper leaves at full flowering stage, according to the method described by Aaron [29]. The maximum photochemical efficiency of photosystem II $(F v / F m)$ was measured using a fluorometer (Model OS-30P).

\subsection{Leaf gas exchange attributes}

Gas exchange factors such as net photosynthetic rate $\left(P_{\mathrm{N}}\right)$, transpiration rate $(E)$ and stomatal conductance $\left(g_{\mathrm{s}}\right)$ were measured using a calibrated portable gas exchange device (HCM-1000, Walz). Measurements were accomplished employing the youngest fully expanded leaf samples under full sunlight during midday between 11:00 and 13:00. All measurements were made at the leaf temperature of $28 \pm 2{ }^{\circ} \mathrm{C}, \mathrm{CO}_{2}$ concentration of $370-390 \mathrm{mg} \mathrm{kg}^{-1}$ and photosynthetic photon flux density (PPFD) of 350-500 $\mu \mathrm{mol} \mathrm{m} \mathrm{m}^{-2} \mathrm{~s}^{-1}$.

\subsection{Electrolyte leakage (EL), malondialdehyde (MDA), hydrogen peroxide $\left(\mathrm{H}_{2} \mathrm{O}_{2}\right)$ and proline concentrations (oxidative stress biomarkers)}

Measuring electrolyte leakage (EL \%) was performed applying a conductivity meter according to Ozden et al. [30]. The content of MDA was assessed by the 
thiobarbituric acid (TBA) according to Wang et al. [23] and was calculated on a fresh weight basis, using the following formula:

$\left(\mathrm{nmolMDAg}{ }^{-1}\right)=6.54(\mathrm{OD} 532-\mathrm{OD} 600)-0.56(\mathrm{OD} 450) \times 1000$

$\mathrm{H}_{2} \mathrm{O}_{2}$ was measured according to the method described by Velikova and Loreto [31]. Proline content was measured in fully expanded upper leaves using the method of Bates et al. [32]. The proline content was determined from a standard curve and expressed as $\mu \mathrm{mol}$ proline $\mathrm{g}^{-1} \mathrm{FW}$.

\subsection{Estimation of enzymatic antioxidant activities and the ascorbate-glutathione cycle}

Fresh leaf samples of black cumin seedlings were homogenized with $1 \mathrm{~mL}$ of ice-cold $100 \mathrm{mmol}$ potassium phosphate buffer ( $\mathrm{pH} 7.0$ ) containing $1 \%$ of polyvinylpyrrolidone. The homogenate was centrifuged at $11,500 \times g$ for $30 \mathrm{~min}$ at $4{ }^{\circ} \mathrm{C}$, and its supernatant was used to measure different enzyme activities. CAT (CAT: 1.11.1.6) activity was measured according to the method described by Hasanuzzaman et al. [33]. Superoxide dismutase (SOD, EC1.15.1.1) activity was estimated by recording the reduction in absorbance of superoxide-nitroblue tetrazolium complex by the enzyme [34]. Ascorbate peroxidase (APX, 1.11.1.11) activity was measured according to Nakano and Asada [35]. Glutathione reductase (GR, 1.6.4.2) activity was measured according to Hasanuzzaman et al. [33]. Glutathione peroxidase (QE 1.11.1.9) activity was determined according to the method described by Lopez-Vargas et al. [36]. Glutathione (GSH) contents were measured according to Hasanuzzaman et al. [33].

\subsection{Extraction of total lipids}

\subsubsection{Fatty acids content}

The seeds $(10 \mathrm{~g})$ were powdered by electric milling and mixed with $50 \mathrm{~mL}$ petroleum-ether solvents $\left(40-60^{\circ} \mathrm{C}\right)$ for $8 \mathrm{~h}$ in a Soxhlet apparatus according to the experimental method of Khalid [30] with little modification. To obtain the total lipid contents (\%) and lipid yield ( g plant $^{-1}$ ), oil was then removed after mixture filtration and solvent evaporation under reduced pressure and temperature.

\subsubsection{Fatty acid composition}

For detection of fatty acids composition, the gas chromatography was accomplished. Fatty acids methyl esters were prepared by adding $100 \mu \mathrm{l}$ of $\mathrm{CH}_{3} \mathrm{NaO}(0.5 \mathrm{~mol})$ to $50 \mu \mathrm{l}$ of seed-extracted oil and dissolved in $1 \mathrm{ml}$ of $\mathrm{n}$-hexane, and the mixture was shaken for $15 \mathrm{~min}$ at room temperature. The final solution was added by sodium sulfate according to Goli et al. [37]. A $100 \mathrm{~m}$ capillary column (HP-88) was used to separate fatty acids methyl esters. The temperature schedule for this investigation was $175^{\circ} \mathrm{C}$ (1 min) as initial temperature following with $2.5^{\circ} \mathrm{C} \mathrm{min}{ }^{-1}$ increasing to $240^{\circ} \mathrm{C}$ which lasted $26 \mathrm{~min} ; 250^{\circ} \mathrm{C}$ was considered for both the flame ionization detector and injector temperature. The carrier gas was nitrogen, and samples were injected with a 50:1 split ratio.

\subsection{Statistical analysis}

All the data were processed using analysis of variance (ANOVA), and means were then separated by the LSD test using SAS (version 8.2; SAS Institute, Cary, NC, USA) software.

\section{Results}

\subsection{Seed and oil yield of karrikin- and $\mathrm{CaCl}_{2}$-treated black cumin plants under salt stress}

When no salinity stress was imposed and without foliar application of calcium, seed yield was $14.43 \%$ higher in plants grown with karrikin-treated black cumin plants than those not receiving the karrikin pretreatment (controls), although changes of oil content and oil yield under the same conditions were not significant (Table 1). The seed yield showed a significant decline with increasing $\mathrm{NaCl}$ concentration (Table 1). The oil content and oil yield were initially elevated with increasing $\mathrm{NaCl}$ level up to $50 \mathrm{mmol}$ but higher increase in salt concentration (above $50 \mathrm{mmol}$ ) was associated with significant decrease (Table 1). Although calcium foliar application in nonstressed plants did not result in a significant alteration of seed yield, oil content and oil yield, by imposing salinity stress, calcium application significantly alleviated the negative effects of $\mathrm{NaCl}$ toxicity on the above-mentioned traits (Table 1).

\subsection{Changes in unsaturated and saturated fatty acids in karrikin- and $\mathrm{CaCl}_{2}$-treated black cumin plants under salt stress}

A higher proportion of unsaturated fatty acids (UFAs) (65.25\%) than saturated fatty acids (SFAs) $(29.48 \%)$ was observed in the seeds of black cumin plants grown under nonsaline conditions in the absence of karrikin and calcium treatments (Tables 2 and 3 ). When no salinity stress was imposed and in the absence of karrikin and calcium treatments, the most important saturated fatty acid component of black cumin plants was characterized by palmitic acid (29.51\%) (Table 2 ) and unsaturated fatty acid reigned 
Table 1 Seed yield $\left(\right.$ g plant $\left.^{-1}\right)$, oil content (\%) and oil yield (g plant ${ }^{-1}$ ) means comparison against salinity, as affected by karrikin pretreatment and foliar application of $\mathrm{CaCl}_{2}$

\begin{tabular}{|c|c|c|c|c|c|}
\hline Karrikin $(10 \mu \mathrm{mol})$ & $\mathrm{NaCl}(\mathrm{mmol})$ & $\mathrm{CaCl}_{2}(\mathrm{mmol})$ & $\begin{array}{l}\text { Seed yield } \\
\left(\text { g plant }^{-1}\right)\end{array}$ & Oil content (\%) & $\begin{array}{l}\text { Oil yield } \\
\left(\text { g plant }^{-1}\right)\end{array}$ \\
\hline \multirow[t]{12}{*}{ Karrikin pretreatment } & \multirow[t]{3}{*}{0} & 0 & $3.95^{\mathrm{b}}$ & $17.85^{\text {cde }}$ & $2.62^{\mathrm{cd}}$ \\
\hline & & 50 & $4.60^{\mathrm{a}}$ & $19.38^{c}$ & $2.89^{c}$ \\
\hline & & 100 & $4.73^{\mathrm{a}}$ & $19.59^{c}$ & $2.81^{\mathrm{cd}}$ \\
\hline & \multirow[t]{3}{*}{50} & 0 & $3.55^{\text {cde }}$ & $22.90^{b}$ & $3.77^{\mathrm{ab}}$ \\
\hline & & 50 & $3.87^{b c}$ & $25.78^{\mathrm{a}}$ & $4.18^{a}$ \\
\hline & & 100 & $3.84^{b c}$ & $25.80^{\mathrm{a}}$ & $4.00^{\mathrm{ab}}$ \\
\hline & \multirow[t]{3}{*}{100} & 0 & $2.57^{\mathrm{hi}}$ & $15.98^{\mathrm{ef}}$ & $2.25^{\mathrm{de}}$ \\
\hline & & 50 & $3.13^{\mathrm{fg}}$ & $16.55^{\text {def }}$ & $2.58^{\mathrm{cd}}$ \\
\hline & & 100 & $3.07^{\mathrm{fg}}$ & $16.68^{\text {def }}$ & $2.66^{\mathrm{cd}}$ \\
\hline & \multirow[t]{3}{*}{150} & 0 & $1.62^{\prime}$ & $11.29^{\text {hi }}$ & $1.53^{\mathrm{fg}}$ \\
\hline & & 50 & $2.36^{\mathrm{ij}}$ & $12.92^{\mathrm{gh}}$ & $1.94^{\mathrm{ef}}$ \\
\hline & & 100 & $2.65^{\mathrm{hi}}$ & $13.25^{\mathrm{gh}}$ & $2.33^{\text {cde }}$ \\
\hline \multirow[t]{12}{*}{ Nonkarrikin pretreatment } & \multirow[t]{3}{*}{0} & 0 & $3.38^{\mathrm{def}}$ & $18.53^{\mathrm{cd}}$ & $2.83^{\mathrm{cd}}$ \\
\hline & & 50 & $3.91^{b c}$ & $18.61^{\mathrm{cd}}$ & $2.78^{\mathrm{cd}}$ \\
\hline & & 100 & $3.74^{\mathrm{bcd}}$ & $19.26^{c}$ & $2.59^{\mathrm{cd}}$ \\
\hline & \multirow[t]{3}{*}{50} & 0 & $2.89^{\mathrm{gh}}$ & $21.86^{\mathrm{b}}$ & $3.54^{\mathrm{b}}$ \\
\hline & & 50 & $3.16^{\mathrm{fg}}$ & $23.08^{b}$ & $3.89^{\mathrm{ab}}$ \\
\hline & & 100 & $3.37^{\mathrm{ef}}$ & $23.43^{b}$ & $3.99^{\mathrm{ab}}$ \\
\hline & \multirow[t]{3}{*}{100} & 0 & $2.13^{\mathrm{jk}}$ & $14.69^{f g}$ & $1.74^{\text {ef }}$ \\
\hline & & 50 & $2.69^{\mathrm{hi}}$ & $16.56^{\text {def }}$ & $2.65^{\mathrm{cd}}$ \\
\hline & & 100 & $2.68^{\mathrm{hi}}$ & $16.68^{\text {def }}$ & $2.92^{C}$ \\
\hline & \multirow[t]{3}{*}{150} & 0 & $1.19^{\mathrm{m}}$ & $10.16^{i}$ & $1.09^{g}$ \\
\hline & & 50 & $1.79^{\mathrm{kl}}$ & $12.20^{\mathrm{hi}}$ & $1.51^{\mathrm{fg}}$ \\
\hline & & 100 & $1.81^{\mathrm{kl}}$ & $12.42^{\mathrm{h}}$ & $1.44^{\mathrm{fg}}$ \\
\hline \multicolumn{3}{|l|}{$\operatorname{LSD}_{(0.05)}$} & 0.37 & 2.21 & 0.59 \\
\hline
\end{tabular}

Values (means of three replicates) with different superscripts are significantly different by the LSD multiple range test at $P \leq 0.05$ by petroselinic acid (47.16\%) and linoleic acid (12.94\%) (Table 3). However, under salinity stress, total saturated fatty acids (Table 2) and total unsaturated fatty acids (Table 3) increased and decreased, respectively, in plants that were not under salt stress. It is interesting to note that karrikin pretreatment of black cumin seeds resulted in a decrease in the total amount of saturated fatty acids (Table 2) and increase in total unsaturated fatty acids (Table 3) compared to plants that were only under salinity stress. However, foliarapplied calcium in karrikin-pretreated plants under salinity stress resulted in greater reduction of saturated fatty acids and greater increase in unsaturated fatty acids compared to $\mathrm{NaCl}$ alone (Tables 2 and 3).

\subsection{Chlorophyll content, Fv/Fm ratio and photosynthesis parameters of karrikin- and $\mathrm{CaCl} 2$-treated black cumin plants under salt stress}

Total chlorophyll content, Fv/Fm ratio, net photosynthetic rate $\left(P_{N}\right)$, transpiration rate $(E)$ and stomatal conductance $\left(g_{s}\right)$ of $\mathrm{NaCl}$-treated plants were decreased compared to control (Table 4). Karrikin pretreatment followed by calcium foliar application improved all above-mentioned traits of $\mathrm{NaCl}$-treated black cumin plants compared to $\mathrm{NaCl}$ alone (Table 4).

\subsection{Electrolyte leakage, proline content, $\mathrm{H}_{2} \mathrm{O}_{2}$ and MDA accumulation in karrikin- and $\mathrm{CaCl}_{2}$-treated black cumin plants under salt stress}

Exposure to salt stress was associated with increased electrolyte leakage, proline content, $\mathrm{H}_{2} \mathrm{O}_{2}$ production and MDA accumulation. However, pretreatment with karrikin further enhanced proline accumulation while lowered $E L$, MDA accumulation and $\mathrm{H}_{2} \mathrm{O}_{2}$ production in $\mathrm{NaCl}$-stressed black cumin plants relative to $\mathrm{NaCl}$ alone. And interestingly, complementing karrikin trimmed seeds with calcium spraying exacerbated the positive effects of karrikin (Table 5). 
Table 2 Saturated fatty acid compositions (\%) and total saturated fatty acids (TSFAs) (\%) means comparison against salinity, as affected by karrikin pretreatment and foliar application of $\mathrm{CaCl}_{2}$

\begin{tabular}{|c|c|c|c|c|c|c|c|c|}
\hline $\begin{array}{l}\text { Karrikin } \\
(10 \mu \mathrm{mol})\end{array}$ & $\mathrm{NaCl}(\mathrm{mmol})$ & $\mathrm{CaCl}_{2}(\mathrm{mmol})$ & $\begin{array}{l}\text { Caprylic acid } \\
\text { (C8:0) }\end{array}$ & $\begin{array}{l}\text { Capric acid } \\
\text { (C10:0) }\end{array}$ & $\begin{array}{l}\text { Lauric acid } \\
\text { (C12:0) }\end{array}$ & $\begin{array}{l}\text { Myristic acid } \\
\text { (C14:0) }\end{array}$ & $\begin{array}{l}\text { Palmitic } \\
\text { acid } \\
(\mathrm{C} 16: 0)\end{array}$ & TSFA (\%) \\
\hline
\end{tabular}

(\%)

\begin{tabular}{|c|c|c|c|c|c|c|c|c|}
\hline \multirow{12}{*}{$\begin{array}{c}\text { Karrikin pre- } \\
\text { treatment }\end{array}$} & \multirow[t]{3}{*}{0} & 0 & $1.17^{\mathrm{fghi}}$ & $1.44^{\text {fghi }}$ & $0.74^{\mathrm{klm}}$ & $0.18^{\mathrm{ij}}$ & $25.94^{\mathrm{h}}$ & $29.48^{j}$ \\
\hline & & 50 & $0.97^{\text {ghij }}$ & $1.12^{i}$ & $0.32^{\mathrm{m}}$ & $0.11^{\mathrm{j}}$ & $21.96^{i}$ & $24.49^{k}$ \\
\hline & & 100 & $0.95^{\mathrm{hij}}$ & $1.10^{\mathrm{i}}$ & $0.33^{m}$ & $0.14^{\mathrm{ij}}$ & $21.94^{i}$ & $24.46^{k}$ \\
\hline & \multirow[t]{3}{*}{50} & 0 & $0.82^{\mathrm{ijk}}$ & $2.18^{\text {bcde }}$ & $1.42^{\mathrm{j}}$ & $0.57^{\text {cdef }}$ & $33.11^{f}$ & $38.10^{\mathrm{h}}$ \\
\hline & & 50 & $0.56^{\mathrm{jk}}$ & $1.50^{\text {efghi }}$ & $1.15^{\mathrm{jk}}$ & $0.31^{\text {fghij }}$ & $30.41^{g}$ & $33.92^{\mathrm{i}}$ \\
\hline & & 100 & $0.42^{\mathrm{k}}$ & $1.22^{\mathrm{hi}}$ & $0.95^{\mathrm{jkl}}$ & $0.28^{g h i j}$ & $29.35^{\mathrm{g}}$ & $32.24^{i}$ \\
\hline & \multirow[t]{3}{*}{100} & 0 & $1.46^{\mathrm{efg}}$ & $2.69^{\mathrm{ab}}$ & $2.74^{\mathrm{fgh}}$ & $0.83^{b c}$ & $38.62^{d}$ & $46.33^{d}$ \\
\hline & & 50 & $1.28^{\mathrm{fghi}}$ & $2.27^{\mathrm{abcd}}$ & $2.44^{\mathrm{hi}}$ & $0.64^{\text {cde }}$ & $35.22^{\mathrm{ef}}$ & $41.85^{\mathrm{fg}}$ \\
\hline & & 100 & $1.24^{\mathrm{fghi}}$ & $2.05^{\text {bcdefg }}$ & $2.23^{\mathrm{hi}}$ & $0.55^{\text {cdefg }}$ & $35.05^{\mathrm{ef}}$ & $41.13^{g}$ \\
\hline & \multirow[t]{3}{*}{150} & 0 & $2.00^{\mathrm{cd}}$ & $2.35^{\mathrm{abc}}$ & $3.23^{\text {def }}$ & $1.17^{\mathrm{a}}$ & $43.08^{\mathrm{ab}}$ & $51.83^{b}$ \\
\hline & & 50 & $1.44^{\text {efgh }}$ & $1.99^{\text {bcdefg }}$ & $2.60^{\mathrm{ghi}}$ & $1.01^{\mathrm{ab}}$ & $38.35^{d}$ & $45.39^{\text {de }}$ \\
\hline & & 100 & $1.28^{\mathrm{fghi}}$ & $1.91^{\text {cdefgh }}$ & $2.34^{\mathrm{hi}}$ & $0.77^{\mathrm{bcd}}$ & $38.01^{d}$ & $44.31^{\mathrm{def}}$ \\
\hline \multirow{12}{*}{$\begin{array}{l}\text { Nonkarrikin pre- } \\
\text { treatment }\end{array}$} & \multirow[t]{3}{*}{0} & 0 & $1.13^{\mathrm{fghi}}$ & $1.58^{\text {defghi }}$ & $0.89^{\mathrm{jkl}}$ & $0.32^{\mathrm{fghij}}$ & $29.51^{\mathrm{g}}$ & $33.42^{i}$ \\
\hline & & 50 & $0.82^{\mathrm{ijk}}$ & $1.14^{i}$ & $0.65^{\mathrm{klm}}$ & $0.23^{h i j}$ & $26.33^{\mathrm{h}}$ & $29.17^{j}$ \\
\hline & & 100 & $0.94^{\mathrm{ij}}$ & $1.21^{\mathrm{hi}}$ & $0.52^{1 \mathrm{~m}}$ & $0.19^{\mathrm{ij}}$ & $25.36^{\mathrm{h}}$ & $28.22^{j}$ \\
\hline & \multirow[t]{3}{*}{50} & 0 & $1.49^{\mathrm{ef}}$ & $1.91^{\text {cdefgh }}$ & $2.65^{\mathrm{ghi}}$ & $0.50^{\text {defgh }}$ & $36.67^{\text {de }}$ & $43.22^{\text {efg }}$ \\
\hline & & 50 & $0.98^{\text {ghij }}$ & $1.41^{\text {ghi }}$ & $2.37^{\mathrm{hi}}$ & $0.32^{\text {fghij }}$ & $33.31^{f}$ & $38.39^{h}$ \\
\hline & & 100 & $1.11^{\mathrm{fghi}}$ & $1.37^{\mathrm{ghi}}$ & $2.12^{i}$ & $0.30^{\text {fghij }}$ & $33.08^{f}$ & $37.99^{h}$ \\
\hline & \multirow[t]{3}{*}{100} & 0 & $2.51^{\mathrm{ab}}$ & $2.24^{\mathrm{abcd}}$ & $3.84^{\mathrm{bc}}$ & $0.57^{\text {cdef }}$ & $41.90^{b}$ & $51.07^{b}$ \\
\hline & & 50 & $1.78^{\text {cde }}$ & $2.16^{\text {bcdef }}$ & $3.39^{\text {cde }}$ & $0.41^{\text {efghi }}$ & $38.96^{\mathrm{cd}}$ & $46.69^{c d}$ \\
\hline & & 100 & $1.52^{\text {def }}$ & $1.83^{\text {cdefghi }}$ & $3.10^{\mathrm{efg}}$ & $0.39^{\text {efghij }}$ & $36.61^{\text {de }}$ & $43.45^{\text {efg }}$ \\
\hline & \multirow[t]{3}{*}{150} & 0 & $2.95^{\mathrm{a}}$ & $2.97^{\mathrm{a}}$ & $4.58^{\mathrm{a}}$ & $0.98^{\mathrm{ab}}$ & $45.13^{\mathrm{a}}$ & $56.61^{\mathrm{a}}$ \\
\hline & & 50 & $2.16^{b c}$ & $1.68^{\text {cdefghi }}$ & $3.99^{b}$ & $0.49^{\text {defgh }}$ & $41.39^{b}$ & $49.71^{b}$ \\
\hline & & 100 & $2.13^{b c}$ & $1.57^{\text {defghi }}$ & $3.75^{\mathrm{bcd}}$ & $0.64^{\text {cde }}$ & $41.19^{b c}$ & $49.28^{b c}$ \\
\hline $\operatorname{LSD}_{(0.05)}$ & & & 0.49 & 0.73 & 0.54 & 0.28 & 2.42 & 2.73 \\
\hline
\end{tabular}

Values (means of three replicates) with different superscripts are significantly different by the LSD multiple range test at $P \leq 0.05$

\subsection{Changes in enzymatic and nonenzymatic antioxidant defense system of karrikin- and $\mathrm{CaCl}_{2}$-treated black cumin plants under salt stress}

Salinity stress had different effects on the activity of the five enzymes investigated in this. The activities of CAT, APX and SOD in salt-affected black cumin plants were higher than nonsalt-affected plants (Table 6). Interestingly, karrikin pretreatment resulted in further enhanced CAT, APX and SOD activities of $\mathrm{NaCl}$-stressed plants compared to $\mathrm{NaCl}$ alone (Table 6) and supplementation of karrikin pretreatment with calcium further increased CAT, APX and SOD activities (Table 6). Salinity exposure resulted in an enhanced GPX activity which declined with karrikin and calcium treatments compared to $\mathrm{NaCl}$ alone (Table 6). Salt stress also reduced the activity of GR compared to control plants. However, pretreatment with karrikin significantly enhanced the activity of $\mathrm{GR}$ in $\mathrm{NaCl}$-stressed plants compared to $\mathrm{NaCl}$ alone (Table 6). Supplementation of calcium to $\mathrm{NaCl}$-stressed black cumin plants further increased the activity of $\mathrm{GR}$ relative to $\mathrm{NaCl}$ alone (Table 6). Salt stress reduced GSH content and GSH/GSSG ratio while increased GSSG content compared to the control (Table 7). However, GSH content increased with karrikin pretreatment and with calcium supplementation in $\mathrm{NaCl}$-stressed seedlings compared to $\mathrm{NaCl}$ alone. The content of GSSG in $\mathrm{NaCl}$-stressed plants declined with karrikin and calcium foliar application compared to $\mathrm{NaCl}$ alone (Table 7).

\subsection{Pearson correlation matrix of traits measured in karrikin- and $\mathrm{CaCl}_{2}$-treated black cumin plants under salt stress}

Pearson's correlation coefficients in Table 8 show the positive relationship between seed yield with total saturated fatty acids, chlorophyll content, $\mathrm{Fv} / \mathrm{Fm}$, net photosynthetic rate, transpiration rate, stomatal conductance, glutathione 
Table 3 Unsaturated fatty acid compositions (\%) and total unsaturated fatty acids (TUFAs) (\%) means comparison against salinity, as affected by karrikin pretreatment and foliar application of $\mathrm{CaCl}_{2}$

\begin{tabular}{|c|c|c|c|c|c|c|c|}
\hline Karrikin $(10 \mu \mathrm{mol})$ & $\mathrm{NaCl}(\mathrm{mmol})$ & $\mathrm{CaCl}_{2}(\mathrm{mmol})$ & $\begin{array}{l}\text { Oleic acid } \\
\text { (C18:1n-9) } \\
(\%)\end{array}$ & $\begin{array}{l}\text { Linoleic acid } \\
(C 18: 2 n-6)\end{array}$ & $\begin{array}{l}\text { Linolenic acid } \\
(C 18: 3 n-3)\end{array}$ & $\begin{array}{l}\text { Petroselinic acid } \\
\text { (C18:1n-12) }\end{array}$ & TUFA (\%) \\
\hline \multirow[t]{12}{*}{ Karrikin pretreatment } & \multirow[t]{3}{*}{0} & 0 & $1.77^{\mathrm{bc}}$ & $12.94^{\mathrm{bc}}$ & $0.62^{b}$ & $49.92^{b}$ & $65.25^{b}$ \\
\hline & & 50 & $1.96^{\mathrm{ab}}$ & $14.26^{\mathrm{ab}}$ & $0.75^{\mathrm{a}}$ & $54.55^{\mathrm{a}}$ & $71.52^{\mathrm{a}}$ \\
\hline & & 100 & $2.06^{\mathrm{a}}$ & $14.96^{\mathrm{a}}$ & $0.76^{\mathrm{a}}$ & $55.14^{\mathrm{a}}$ & $72.93^{\mathrm{a}}$ \\
\hline & \multirow[t]{3}{*}{50} & 0 & $1.41^{\mathrm{de}}$ & $8.28^{\mathrm{g}}$ & $0.50 \mathrm{~b}^{\mathrm{cd}}$ & $43.37^{\mathrm{ef}}$ & $53.56^{\mathrm{e}}$ \\
\hline & & 50 & $1.75^{\mathrm{bc}}$ & $11.23^{\mathrm{de}}$ & $0.59^{b c}$ & $49.40^{b c}$ & $62.97^{b c}$ \\
\hline & & 100 & $2.08^{\mathrm{a}}$ & $11.61^{\text {cde }}$ & $0.61^{\mathrm{b}}$ & $47.08^{c d}$ & $61.39^{c d}$ \\
\hline & \multirow[t]{3}{*}{100} & 0 & $0.60^{\text {hijk }}$ & $5.80^{\mathrm{ijk}}$ & $0.38^{\text {def }}$ & $36.75^{\mathrm{hi}}$ & $43.54^{\mathrm{h}}$ \\
\hline & & 50 & $0.79^{g h i}$ & $7.55^{\mathrm{gh}}$ & $0.44^{\text {def }}$ & $38.88^{\mathrm{ghi}}$ & $47.67^{\mathrm{fg}}$ \\
\hline & & 100 & $0.86^{\mathrm{gh}}$ & $7.82^{\mathrm{gh}}$ & $0.47^{\text {cde }}$ & $39.32^{g h}$ & $48.47^{f}$ \\
\hline & \multirow[t]{3}{*}{150} & 0 & $0.62^{\text {hijk }}$ & $3.93^{\mathrm{mn}}$ & $0.17^{\mathrm{hi}}$ & $33.82^{\mathrm{jk}}$ & $38.54^{i}$ \\
\hline & & 50 & $0.85^{\mathrm{gh}}$ & $5.18^{\mathrm{jklm}}$ & $0.23^{\mathrm{gh}}$ & $36.22^{\mathrm{ij}}$ & $42.47^{\mathrm{h}}$ \\
\hline & & 100 & $0.82^{g h}$ & $5.50^{\mathrm{jkl}}$ & $0.23^{g h}$ & $37.17^{\mathrm{hi}}$ & $43.73^{h}$ \\
\hline \multirow[t]{12}{*}{ Nonkarrikin pretreatment } & \multirow[t]{3}{*}{0} & 0 & $1.28^{\mathrm{ef}}$ & $10.13^{\text {ef }}$ & $0.32^{\mathrm{fg}}$ & $47.16^{\mathrm{bcd}}$ & $58.89^{d}$ \\
\hline & & 50 & $1.63^{\mathrm{cd}}$ & $11.53^{\text {cde }}$ & $0.36^{\mathrm{ef}}$ & $49.58^{b c}$ & $63.10^{b c}$ \\
\hline & & 100 & $1.72^{\mathrm{bc}}$ & $11.70^{\mathrm{cd}}$ & $0.37^{\mathrm{ef}}$ & $49.71^{b c}$ & $63.50^{\mathrm{b}} \mathrm{c}$ \\
\hline & \multirow[t]{3}{*}{50} & 0 & $0.84^{g h}$ & $7.18^{\text {ghi }}$ & $0.38^{\text {def }}$ & $40.80^{\mathrm{fg}}$ & $49.20^{f}$ \\
\hline & & 50 & $1.00^{\mathrm{fg}}$ & $8.65^{\mathrm{fg}}$ & $0.40^{\text {def }}$ & $44.05^{\mathrm{e}}$ & $54.10^{\mathrm{e}}$ \\
\hline & & 100 & $1.25^{\mathrm{ef}}$ & $8.55^{\mathrm{g}}$ & $0.41^{\text {def }}$ & $44.82^{\mathrm{de}}$ & $55.03^{\mathrm{e}}$ \\
\hline & \multirow[t]{3}{*}{100} & 0 & $0.51^{\mathrm{ijk}}$ & $4.73^{\mathrm{klm}}$ & $0.17^{\mathrm{hi}}$ & $33.10^{\mathrm{kl}}$ & $38.50^{i}$ \\
\hline & & 50 & $0.66^{\text {hij }}$ & $6.54^{\mathrm{hij}}$ & $0.21^{\mathrm{gh}}$ & $36.43^{\mathrm{ij}}$ & $43.83^{h}$ \\
\hline & & 100 & $0.84^{g h}$ & $6.47^{\mathrm{hij}}$ & $0.23^{\mathrm{gh}}$ & $37.18^{\mathrm{hi}}$ & $44.71^{\mathrm{gh}}$ \\
\hline & \multirow[t]{3}{*}{150} & 0 & $0.21^{1}$ & $2.81^{n}$ & $0.05^{\mathrm{i}}$ & $27.30^{n}$ & $30.38^{k}$ \\
\hline & & 50 & $0.37^{\mathrm{kl}}$ & $3.73^{\mathrm{mn}}$ & $0.13^{\mathrm{hi}}$ & $29.64^{\mathrm{mn}}$ & $33.86^{j}$ \\
\hline & & 100 & $0.48^{\mathrm{jkl}}$ & $4.24^{\operatorname{Imn}}$ & $0.13^{\mathrm{hi}}$ & $30.47^{1 \mathrm{~m}}$ & $35.33^{\mathrm{ij}}$ \\
\hline \multicolumn{3}{|l|}{$\operatorname{LSD}_{(0.05)}$} & 0.28 & 1.53 & 0.13 & 2.82 & 3.28 \\
\hline
\end{tabular}

Values (means of three replicates) with different superscripts are significantly different by the LSD multiple range test at $P \leq 0.05$

reductase and reduced glutathione/oxidized glutathione ratio $(0.91,0.91,0.93,0.91,0.90,0.93,0.92$ and 0.86 , respectively, $P$-value $=0.0001)$ and also oil yield with total saturated fatty acids, chlorophyll content, Fv/Fm, net photosynthetic rate, transpiration rate, stomatal conductance, glutathione reductase and reduced glutathione/oxidized glutathione ratio $(0.91,0.50,0.63,0.51,0.90,0.93,0.71$ and 0.86 , respectively, $P$-value $=0.001)$, while they show the negative correlation between seed yield and electrolyte leakage, proline content, $\mathrm{H}_{2} \mathrm{O}_{2}$ generation, MDA accumulation, CAT, APX, SOD and GPX activities $(-0.92,-0.93,-0.71,-0.92,-0.88$,$0.70,-0.80$ and -0.74 , respectively, $P$ value $=0.001$ ) as well as oil yield with electrolyte leakage, proline content, $\mathrm{H}_{2} \mathrm{O}_{2}$ generation, MDA accumulation, CAT, APX, SOD and GPX activities $(-0.92,-0.93,-0.71,-0.91,-0.88,-0.32,-0.50,-$ 0.47 , respectively, $P$ value $=0.001$ ). A very interesting point in this study was that there was a significant negative correlation between seed yield and oil yield with total saturated fatty acids ( -0.94 and -0.54 , respectively, $P$ value $=0.001)$, while the relationship between seed yield and oil yield with total unsaturated fatty acids ( 0.91 and 0.91 , respectively $P$ value $=0.001$ ) was significantly positive. Totally, the results showed a greater increase in seed and oil yield of karrikin- and $\mathrm{CaCl}_{2}$-treated black cumin and the beginning of an increasing trend, as the $\mathrm{CaCl}_{2}$ concentration increased (Table 1) which further justified this correlation (Table 8). A statistically significant correlation in the opposite direction was also found related to chlorophyll content, $\mathrm{Fv} / \mathrm{Fm}$ ratio and photosynthetic parameters with electrolyte leakage, $\mathrm{H}_{2} \mathrm{O}_{2}$ generation and MDA accumulation (Table 8). 
Table 4 Chlorophyll content, $F v / F m$ ratio, net photosynthetic rate $\left(P_{\mathrm{N}}\right)$, transpiration rate $(E)$ and stomatal conductance $\left(g_{\mathrm{s}}\right)$ means comparison against salinity, as affected by karrikin pretreatment and foliar application of $\mathrm{CaCl}_{2}$

\begin{tabular}{|c|c|c|c|c|c|c|c|}
\hline Karrikin $(10 \mu \mathrm{mol})$ & $\mathrm{NaCl}(\mathrm{mmol})$ & $\mathrm{CaCl}_{2}(\mathrm{mmol})$ & $\mathrm{Chl}\left(\mathrm{mg} \mathrm{g}^{-1} \mathrm{FW}\right)$ & $\mathrm{Fv} / \mathrm{Fm}$ & $\begin{array}{l}P_{\mathrm{N}}(\mu \mathrm{mol} \\
\left.\mathrm{CO}_{2} \mathrm{~m}^{-2} \mathrm{~s}^{-1}\right)\end{array}$ & $\begin{array}{l}E(\mathrm{mmol} \\
\mathrm{H}_{2} \mathrm{O} \mathrm{m}^{-2} \mathrm{~s}^{-1}\end{array}$ & $G_{\mathrm{S}}\left(\mathrm{mol} \mathrm{m}^{-2} \mathrm{~s}^{-1}\right)$ \\
\hline \multirow[t]{12}{*}{ Karrikin pretreatment } & \multirow[t]{3}{*}{0} & 0 & $2.72^{\mathrm{cd}}$ & $0.81^{\mathrm{ab}}$ & $10.93^{b}$ & $0.60^{\mathrm{ab}}$ & $3.91^{\mathrm{c}}$ \\
\hline & & 50 & $3.32^{\mathrm{ab}}$ & $0.88^{\mathrm{a}}$ & $14.29^{\mathrm{a}}$ & $0.68^{a}$ & $4.87^{\mathrm{a}}$ \\
\hline & & 100 & $3.79^{\mathrm{a}}$ & $0.87^{\mathrm{a}}$ & $14.42^{\mathrm{a}}$ & $0.67^{\mathrm{a}}$ & $4.73^{b}$ \\
\hline & \multirow[t]{3}{*}{50} & 0 & $1.66^{f}$ & $0.61^{d}$ & $9.32^{\mathrm{cd}}$ & $0.53^{\mathrm{abcd}}$ & $2.84^{\mathrm{g}}$ \\
\hline & & 50 & $2.25^{\mathrm{de}}$ & $0.71^{c}$ & $10.90^{\mathrm{b}}$ & $0.61^{\mathrm{ab}}$ & $3.75^{\mathrm{e}}$ \\
\hline & & 100 & $2.30^{\mathrm{de}}$ & 0.73 & $11.85^{\mathrm{b}}$ & $0.63^{\mathrm{ab}}$ & $3.89^{\mathrm{cd}}$ \\
\hline & \multirow[t]{3}{*}{100} & 0 & $0.82^{\mathrm{ijk}}$ & $0.44^{\mathrm{fg}}$ & $7.13^{\mathrm{fg}}$ & $0.39^{\text {defg }}$ & $1.88^{\mathrm{j}}$ \\
\hline & & 50 & $1.50^{\text {fgh }}$ & $0.51^{\mathrm{ef}}$ & $8.44^{\text {def }}$ & $0.48^{\mathrm{bcd}}$ & $2.26^{i}$ \\
\hline & & 100 & $1.54^{\mathrm{fg}}$ & $0.54^{\mathrm{de}}$ & $8.89^{\text {cde }}$ & $0.50^{\mathrm{bcd}}$ & $2.42^{\mathrm{h}}$ \\
\hline & \multirow[t]{3}{*}{150} & 0 & $0.44^{\mathrm{kl}}$ & $0.24^{\mathrm{jk}}$ & $6.08^{\mathrm{gh}}$ & $0.27^{\text {fgh }}$ & $1.16^{\mathrm{m}}$ \\
\hline & & 50 & $0.87^{\mathrm{ijk}}$ & $0.34^{\mathrm{hi}}$ & $7.33^{\mathrm{fg}}$ & $0.41^{\text {defg }}$ & $1.89^{j}$ \\
\hline & & 100 & $0.92^{\mathrm{ijk}}$ & $0.37^{\mathrm{gh}}$ & $7.32^{\mathrm{fg}}$ & $0.42^{\text {cdef }}$ & $1.91^{\mathrm{j}}$ \\
\hline \multirow[t]{12}{*}{ Nonkarrikin pretreatment } & \multirow[t]{3}{*}{0} & 0 & $1.96^{\mathrm{ef}}$ & $0.73^{\mathrm{bc}}$ & $8.25^{\text {def }}$ & $0.50^{\mathrm{bcd}}$ & $3.24^{f}$ \\
\hline & & 50 & $2.82^{\mathrm{C}}$ & $0.77^{\mathrm{bc}}$ & $10.42^{\mathrm{bc}}$ & $0.58^{\mathrm{abc}}$ & $3.75^{\mathrm{e}}$ \\
\hline & & 100 & $2.94^{\mathrm{bc}}$ & $0.77^{\mathrm{bc}}$ & $10.42^{b c}$ & $0.61^{\mathrm{ab}}$ & $3.81^{\mathrm{de}}$ \\
\hline & \multirow[t]{3}{*}{50} & 0 & $1.10^{\text {ghi }}$ & $0.49^{\mathrm{ef}}$ & $7.27^{f g}$ & $0.41^{\text {defg }}$ & $1.79^{k}$ \\
\hline & & 50 & $1.81^{\mathrm{ef}}$ & $0.62^{d}$ & $7.82^{\text {def }}$ & $0.47^{\text {bcde }}$ & $2.31^{i}$ \\
\hline & & 100 & $1.81^{\mathrm{ef}}$ & $0.62^{d}$ & $8.17^{\text {def }}$ & $0.49^{\text {bcd }}$ & $2.45^{\mathrm{h}}$ \\
\hline & \multirow[t]{3}{*}{100} & 0 & $0.57^{\mathrm{jkl}}$ & $0.26^{\mathrm{ijk}}$ & $5.51^{\mathrm{hi}}$ & $0.25^{\mathrm{gh}}$ & $0.86^{n}$ \\
\hline & & 50 & $0.88^{\mathrm{ijk}}$ & $0.32^{\mathrm{hij}}$ & $7.40^{\text {efg }}$ & $0.31^{\text {efgh }}$ & $1.30^{\prime}$ \\
\hline & & 100 & $1.01^{\mathrm{hij}}$ & $0.34^{\mathrm{hi}}$ & $7.73^{\text {ef }}$ & $0.31^{\mathrm{efgh}}$ & $1.31^{1}$ \\
\hline & \multirow[t]{3}{*}{150} & 0 & $0.17^{1}$ & $0.14^{\prime}$ & $4.39^{i}$ & $0.16^{\mathrm{h}}$ & $0.35^{\mathrm{p}}$ \\
\hline & & 50 & $0.52^{\mathrm{jkl}}$ & $0.21^{\mathrm{kl}}$ & $5.48^{\mathrm{hi}}$ & $0.28^{\mathrm{fgh}}$ & $0.72^{\circ}$ \\
\hline & & 100 & $0.54^{\mathrm{jkl}}$ & $0.23^{k}$ & $5.85^{\mathrm{ghi}}$ & $0.26^{\mathrm{fgh}}$ & $0.76^{\circ}$ \\
\hline \multicolumn{3}{|l|}{$\operatorname{LSD}_{(0.05)}$} & 0.49 & 0.09 & 1.56 & 0.16 & 0.09 \\
\hline
\end{tabular}

Values (means of three replicates) with different superscripts are significantly different by the LSD multiple range test at $P \leq 0.05$

\section{Discussion}

\subsection{Treatment of black cumin with karrikin and $\mathrm{CaCl}_{2}$ ameliorates salinity by enhancing seed and oil yield}

This study aimed to understand the physiological processes involved in black cumin salt tolerance and to induce such processes using karrikin pretreatment and foliarapplied calcium to soothe the damage caused by salinity in photosynthesis and its oxidative stress. Increasing salinity levels caused a noticeable inhibitory impact on seed yield per plant of black cumin, and this decline was more pronounced in the higher levels ( 100 and $150 \mathrm{mmol} \mathrm{NaCl}$ ) compared to the controls. The negative effect of salt stress on seed yield has been reported earlier in several plants such as cumin [38], Foeniculum vulgare [39] and Trachyspermum ammi [40] which further supported our results. Inefficient photosynthesis is one of the reasons for the decrease in seed yield under the conditions of salinity due to the closure of stomata that limit carbon dioxide capture [41]. Our results showed that both karrikin and calcium, either alone or in combination, improved seed yield and seed oil content of black cumin. Calcium application has also alleviated the harmful effects of $\mathrm{NaCl}$ toxicity in species such as Linum usitatissimum [20], rice [42], pistachio [43] and cumin [38]. The decrease in seed oil yield due to salinity could be attributed to inhibition of lipid biosynthesis or stimulation of lipolytic activity and peroxidation [44].

\subsubsection{Treatment of black cumin with karrikin and $\mathrm{CaCl}_{2}$ ameliorates salinity stress by improving the oil quality}

Fatty acids, which are key components of plasma membrane lipids, result in an enhanced salinity tolerance in plants by maintaining membrane fluidity [45]. The effective variations of fatty acids of black cumin seeds in our experiment were also consistent with the results in another cumin seeds [38], further supporting these results. 
Table 5 Electrolyte leakage, proline content, $\mathrm{H}_{2} \mathrm{O}_{2}$ generation and MDA accumulation means comparison against salinity, as affected by karrikin pretreatment and foliar application of $\mathrm{CaCl}_{2}$

\begin{tabular}{|c|c|c|c|c|c|c|}
\hline Karrikin $(10 \mu \mathrm{mol})$ & $\mathrm{NaCl}(\mathrm{mmol})$ & $\mathrm{CaCl}_{2}(\mathrm{mmol})$ & EL (\%) & $\begin{array}{l}\text { Proline } \\
\left(\mu \mathrm{mol} \mathrm{g}{ }^{-1} \mathrm{FW}\right)\end{array}$ & $\begin{array}{l}\mathrm{H}_{2} \mathrm{O}_{2} \\
(\mathrm{nmol} \mathrm{g} \\
-1\end{array}$ & $\begin{array}{l}\text { MDA } \\
\left(\mathrm{nmol} \mathrm{g}^{-1} \mathrm{FW}\right)\end{array}$ \\
\hline \multirow[t]{12}{*}{ Karrikin pretreatment } & \multirow[t]{3}{*}{0} & 0 & $13.80^{\mathrm{j}}$ & $41.81^{1}$ & $2.73^{\mathrm{kl}}$ & $1.91^{\mathrm{klm}}$ \\
\hline & & 50 & $12.72^{\mathrm{j}}$ & $42.34^{1}$ & $2.45^{1}$ & $1.45^{\mathrm{m}}$ \\
\hline & & 100 & $12.04^{j}$ & $42.58^{l}$ & $2.53^{1}$ & $1.48^{\mathrm{m}}$ \\
\hline & \multirow[t]{3}{*}{50} & 0 & $23.77^{\mathrm{h}}$ & $86.44^{\mathrm{hi}}$ & $5.46^{\mathrm{hi}}$ & $2.44^{\mathrm{jkl}}$ \\
\hline & & 50 & $18.77^{i}$ & $90.58^{g h}$ & $4.49^{\mathrm{ijk}}$ & $1.91^{\mathrm{klm}}$ \\
\hline & & 100 & $18.57^{i}$ & $90.67^{\text {gh }}$ & $4.55^{\mathrm{ij}}$ & $1.79^{\mathrm{lm}}$ \\
\hline & \multirow[t]{3}{*}{100} & 0 & $43.26^{\mathrm{e}}$ & $122.73^{f}$ & $8.73^{\text {ef }}$ & $4.28^{f g}$ \\
\hline & & 50 & $37.28^{f}$ & $140.41^{\mathrm{e}}$ & $8.07^{f g}$ & $3.25^{\text {hij }}$ \\
\hline & & 100 & $34.57^{\mathrm{fg}}$ & $140.56^{\mathrm{e}}$ & $8.89^{\text {ef }}$ & $3.37^{\mathrm{hi}}$ \\
\hline & \multirow[t]{3}{*}{150} & 0 & $74.27^{\mathrm{ab}}$ & $170.16^{c}$ & $12.41^{\mathrm{bc}}$ & $6.02^{\mathrm{bcd}}$ \\
\hline & & 50 & $69.23^{c}$ & $191.08^{b}$ & $10.37^{\text {de }}$ & $4.94^{\mathrm{ef}}$ \\
\hline & & 100 & $68.40^{c}$ & $196.54^{\mathrm{a}}$ & $9.23^{\mathrm{ef}}$ & 4.82 \\
\hline \multirow[t]{12}{*}{ Nonkarrikin pretreatment } & \multirow[t]{3}{*}{0} & 0 & $15.45^{\mathrm{ij}}$ & $42.53^{\prime}$ & $3.31^{\mathrm{jkl}}$ & $2.68^{\mathrm{ijk}}$ \\
\hline & & 50 & $15.45^{\mathrm{ij}}$ & $41.21^{1}$ & $2.77^{\mathrm{jkl}}$ & $1.95^{\mathrm{klm}}$ \\
\hline & & 100 & $15.67^{\mathrm{ij}}$ & $41.81^{l}$ & $2.75^{\mathrm{kl}}$ & $1.96^{\mathrm{klm}}$ \\
\hline & \multirow[t]{3}{*}{50} & 0 & $30.80^{\mathrm{g}}$ & $65.37^{\mathrm{k}}$ & $6.66^{\mathrm{gh}}$ & $3.91^{\mathrm{gh}}$ \\
\hline & & 50 & $24.87^{\mathrm{h}}$ & $79.67^{j}$ & $5.39^{\text {hi }}$ & $3.67^{\mathrm{gh}}$ \\
\hline & & 100 & $25.03^{\mathrm{h}}$ & $82.50^{\mathrm{ij}}$ & $5.59^{\text {hi }}$ & $3.70^{\mathrm{gh}}$ \\
\hline & \multirow[t]{3}{*}{100} & 0 & $54.29^{d}$ & $91.67^{9}$ & $11.62^{\text {cd }}$ & $5.84^{\mathrm{cd}}$ \\
\hline & & 50 & $43.56^{\mathrm{e}}$ & $117.86^{f}$ & $10.35^{\mathrm{de}}$ & $5.54^{\text {de }}$ \\
\hline & & 100 & $44.36^{e}$ & $118.39^{f}$ & $10.42^{\text {de }}$ & $5.37^{\text {de }}$ \\
\hline & \multirow[t]{3}{*}{150} & 0 & $78.32^{\mathrm{a}}$ & $119.84^{f}$ & $15.53^{\mathrm{a}}$ & $7.19^{a}$ \\
\hline & & 50 & $70.72^{b c}$ & $153.02^{d}$ & $13.40^{\mathrm{bc}}$ & $6.45^{\mathrm{abc}}$ \\
\hline & & 100 & $70.86^{b c}$ & $153.92^{d}$ & $13.43^{b}$ & $6.73^{\mathrm{ab}}$ \\
\hline \multicolumn{3}{|l|}{$\operatorname{LSD}_{(0.05)}$} & 4.24 & 4.93 & 1.78 & 0.84 \\
\hline
\end{tabular}

Values (means of three replicates) with different superscripts are significantly different by the LSD multiple range test at $P \leq 0.05$

In addition, salinity also lowered the percentage of unsaturated fatty acids so that the degree of this decrease was strongly in accordance with the intensity of salinity. However, knowledge on the role of KARs and calcium both in combination and separately in the composition and amount of fatty acids due is still incomplete.

Treatment of black cumin with karrikin and $\mathrm{CaCl} 2$ ameliorates salinity stress by improving the physiobiochemical attributes of black cumin under salinity stress.

The enhanced photosynthesis and seed yield with calcium application under salt stress with synchronous increase in stomatal conductance and intercellular $\mathrm{CO}_{2}$ concentration demonstrate that the efficiency of $\mathrm{CO}_{2}$ utilization is optimized by using calcium treatment under the conditions of salinity stress [46]. The reduction in leaf gas exchange parameters due to $\mathrm{NaCl}$ toxicity in the present study agrees with similar studies on maize [47], cucumber [16] and tomato plants [24], which further supported our results in black cumin. There is also a concordant report [48] with our results on the positive impact of calcium on improvement in gas exchange parameters in Zoysia japonica under water stress. In support of our results, previous investigation showed that calcium application resulted in an enhanced salinity stress tolerance of Cakile maritima [22] and Nitraria tangutorum [25]. When plants are exposed to salinity stress, they counteract a significant increase in some smolite salts such as proline against the damaging effects of salinity [16]. Proline has also been reported to reduce oxidative stress by counteracting the negative effects of free radicals and increasing the activity of enzymes involved in ROS scavenging [49]. Strigolactones (SLs) and karrikins (KARs) are both a chemical structure of butenolide family whose application in plants improves growth especially under stress conditions [50, 51]. Smith [52] was managed to refine KARs from burnt plant materials and reported that it improved seed germination. Based on the findings of this researcher, we pretreated black cumin seeds with KARs before salinity treatment and then foliar-applied calcium to improve salinity resistance of black cumin plants. When black cumin seeds 
Table 6 Catalase (CAT), ascorbate peroxidase (APX), superoxide dismutase (SOD), guaiacol peroxidase (GPX) and glutathione reductase (GR) activities means comparison against salinity, as affected by karrikin pretreatment and foliar application of $\mathrm{CaCl}_{2}$

\begin{tabular}{|c|c|c|c|c|c|c|c|}
\hline $\begin{array}{l}\text { Karrikin } \\
(10 \mu \mathrm{mol})\end{array}$ & $\begin{array}{l}\mathrm{NaCl} \\
(\mathrm{mmol})\end{array}$ & $\begin{array}{l}\mathrm{CaCl}_{2} \\
(\mathrm{mmol})\end{array}$ & $\begin{array}{l}\text { CAT activity } \\
\left(\mu \mathrm{mol} \mathrm{min}^{-1} \mathrm{mg}^{-1}\right. \\
\text { protein) }\end{array}$ & $\begin{array}{l}\text { APX activity } \\
\left(\mu \mathrm{mol} \mathrm{min}^{-1} \mathrm{mg}^{-1}\right. \\
\text { protein) }\end{array}$ & $\begin{array}{l}\text { SOD activity } \\
\left(\mu \mathrm{mol} \mathrm{min}^{-1} \mathrm{mg}^{-1}\right. \\
\text { protein) }\end{array}$ & $\begin{array}{l}\text { GPX activity } \\
\left(\mu \mathrm{mol} \mathrm{min}^{-1} \mathrm{mg}^{-1}\right. \\
\text { protein) }\end{array}$ & $\begin{array}{l}\text { GR activity } \\
\left(\mu \mathrm{mol} \mathrm{min}^{-1} \mathrm{mg}^{-1}\right. \\
\text { protein) }\end{array}$ \\
\hline \multirow{12}{*}{$\begin{array}{l}\text { Karrikin } \\
\text { pretreat- } \\
\text { ment }\end{array}$} & \multirow[t]{3}{*}{0} & 0 & $0.35^{j}$ & $0.53^{k}$ & $4.52^{\mathrm{h}}$ & $0.56^{\mathrm{i}}$ & $30.63^{\mathrm{ab}}$ \\
\hline & & 50 & $0.43^{j}$ & $0.62^{k}$ & $4.73^{h}$ & $0.50^{i}$ & $31.86^{\mathrm{a}}$ \\
\hline & & 100 & $0.43^{j}$ & $0.64^{k}$ & $4.79^{h}$ & $0.52^{i}$ & $31.66^{\mathrm{a}}$ \\
\hline & \multirow[t]{3}{*}{50} & 0 & $1.03^{i}$ & $1.86^{\mathrm{hi}}$ & $8.29^{g}$ & $2.24^{h}$ & $26.48^{\text {def }}$ \\
\hline & & 50 & $1.22^{\mathrm{ghi}}$ & $2.54^{\mathrm{efg}}$ & $10.03^{\mathrm{fg}}$ & $1.84^{h}$ & $30.71^{\mathrm{ab}}$ \\
\hline & & 100 & $1.36^{\mathrm{fghi}}$ & $2.45^{\text {efgh }}$ & $9.60^{f g}$ & $1.86^{\mathrm{h}}$ & 31.04 \\
\hline & \multirow[t]{3}{*}{100} & 0 & $1.15^{\mathrm{hi}}$ & $2.81^{\text {def }}$ & $12.15^{d}$ & $4.0^{\text {def }}$ & $20.69^{h i}$ \\
\hline & & 50 & $1.57^{\text {efg }}$ & $3.01^{\text {de }}$ & $13.19^{c d}$ & $3.16^{\mathrm{fg}}$ & $24.03^{f g}$ \\
\hline & & 100 & $1.75^{\mathrm{def}}$ & $3.39^{\mathrm{cd}}$ & $13.31^{\mathrm{cd}}$ & $3.21^{\mathrm{efg}}$ & $25.02^{\mathrm{efg}}$ \\
\hline & \multirow[t]{3}{*}{150} & 0 & $2.03^{b c d}$ & $4.13^{\mathrm{ab}}$ & $15.47^{b}$ & $5.95^{\mathrm{ab}}$ & $13.90^{\prime}$ \\
\hline & & 50 & $2.41^{\mathrm{ab}}$ & $4.75^{a}$ & $17.46^{\mathrm{a}}$ & $4.59^{\mathrm{cd}}$ & $19.45^{i j}$ \\
\hline & & 100 & $2.56^{\mathrm{a}}$ & $4.78^{a}$ & $18.16^{\mathrm{a}}$ & $5.21^{b c}$ & $19.48^{i j}$ \\
\hline \multirow{12}{*}{$\begin{array}{l}\text { Nonkar- } \\
\text { rikin } \\
\text { pretreat- } \\
\text { ment }\end{array}$} & \multirow[t]{3}{*}{0} & 0 & $0.34^{j}$ & $0.55^{k}$ & $4.89^{h}$ & $0.58^{i}$ & $27.63^{\text {cde }}$ \\
\hline & & 50 & $0.47^{j}$ & $0.53^{k}$ & $5.29^{h}$ & $0.60^{i}$ & $29.62^{\mathrm{abc}}$ \\
\hline & & 100 & $0.45^{j}$ & $0.59^{k}$ & $5.30^{\mathrm{h}}$ & $0.61^{i}$ & $30.31^{\mathrm{ab}}$ \\
\hline & \multirow[t]{3}{*}{50} & 0 & $0.97^{i}$ & $1.13^{\mathrm{jk}}$ & $8.30^{\mathrm{g}}$ & $2.37^{\mathrm{gh}}$ & $22.82^{g h}$ \\
\hline & & 50 & $1.47^{\text {efgh }}$ & $1.76^{\mathrm{ij}}$ & $10.29^{\text {ef }}$ & $1.83^{h}$ & $28.46^{\mathrm{bcd}}$ \\
\hline & & 100 & $1.84^{\text {cde }}$ & $2.08^{\mathrm{ghi}}$ & $12.08^{\mathrm{de}}$ & $1.88^{\mathrm{h}}$ & $28.75^{\mathrm{bcd}}$ \\
\hline & \multirow[t]{3}{*}{100} & 0 & $1.64^{\mathrm{def}}$ & $2.31^{\text {fghi }}$ & $13.29^{\mathrm{cd}}$ & $4.64^{\mathrm{cd}}$ & $14.96^{1}$ \\
\hline & & 50 & $2.35^{\mathrm{ab}}$ & $3.03^{\mathrm{de}}$ & $14.36^{\mathrm{bc}}$ & $4.07^{\mathrm{de}}$ & $18.17^{\mathrm{ij}}$ \\
\hline & & 100 & $2.41^{\mathrm{ab}}$ & $3.29^{\mathrm{cd}}$ & $14.61^{b c}$ & $3.45^{\mathrm{ef}}$ & $18.75^{\mathrm{ij}}$ \\
\hline & \multirow[t]{3}{*}{150} & 0 & $2.23^{\mathrm{abc}}$ & $3.75^{\mathrm{bc}}$ & $15.48^{\mathrm{b}}$ & $6.27^{a}$ & $10.83^{\mathrm{m}}$ \\
\hline & & 50 & $2.33^{\mathrm{ab}}$ & $4.53^{\mathrm{a}}$ & $19.00^{\mathrm{a}}$ & $5.22^{\mathrm{bc}}$ & $15.26^{\mathrm{kl}}$ \\
\hline & & 100 & $2.17^{\mathrm{abc}}$ & $4.37^{\mathrm{ab}}$ & $19.30^{\mathrm{a}}$ & $5.08^{b c}$ & $17.78^{\mathrm{jk}}$ \\
\hline $\operatorname{LSD}_{(0.05)}$ & & & 0.40 & 0.68 & 1.84 & 0.90 & 2.64 \\
\hline
\end{tabular}

Values (means of three replicates) with different superscripts are significantly different by the LSD multiple range test at $P \leq 0.05$

were pretreated with karrikin followed by calcium foliar application during seedling growth, photosynthetic efficiency under both control and salt stress conditions was observed. Previous researches have provided evidences that show SLs and KARs are involved in the regulation of the plant responses to various abiotic stresses such as salinity and oxidative stresses [53-55]. Application of KARs to $\mathrm{NaCl}$-stressed black cumin plants improved chlorophyll synthesis and gas exchange factors which is perhaps due to its positive role in mitigating salinity stress. These findings are in line with previous reports of Jamil et al. [51] who reported that KARs improved photosynthesis factors of rice plants under the conditions of salinity stress.

\subsubsection{Treatment of black cumin with karrikin and $\mathrm{CaCl}_{2}$ ameliorates salinity by regulating oxidative stress and activating antioxidant defense system}

However, calcium treatment after KARs pretreatment of black cumin seeds enhanced chlorophyll content and the presence of calcium also further enhanced the activity of antioxidant enzymes such as CAT, SOD, APX and GR in black cumin plants, which mitigated the oxidative stress caused by $\mathrm{NaCl}$ toxicity. These results are in accordance with Sunmonu et al. [56] who reported that KARs lowered lipid peroxidation and oxidative stress, thereby improving plant growth under environmental stresses. Similarly, $\mathrm{Ha}$ et al. [57] reported that exogenous SL treatment enhanced drought tolerance of Arabidopsis. In accordance with our findings, KARs have been reported to have a positive impact on declining the accumulation of toxic ions by increasing the accumulation of useful ions such as calcium [51]. Salt stress by exerting osmotic effects on plant causes oxidative stress and production of reactive oxygen species (ROS). These ROS are very harmful for cell membrane permeability and change enzymes activities and the plant photosynthetic system [42]. Our results showed that foliar-applied calcium resulted in the protection of black cumin plants against salinity stress by alleviating the oxidative stress, which was in line with the finding of Xu et al. [46] regarding the positive effect of 
Table 7 Reduced glutathione (GSH), oxidized glutathione (GSSG) and GSH/GSSG ratio means comparison against salinity, as affected by karrikin pretreatment and foliar application of $\mathrm{CaCl}_{2}$

\begin{tabular}{|c|c|c|c|c|c|}
\hline Karrikin $(10 \mu \mathrm{mol})$ & $\mathrm{NaCl}(\mathrm{mmol})$ & $\mathrm{CaCl}_{2}(\mathrm{mmol})$ & $\mathrm{GSH}\left(\mathrm{nmol} \mathrm{g}{ }^{-1} \mathrm{FW}\right)$ & GSSG (nmol g $\left.{ }^{-1} \mathrm{FW}\right)$ & GSH/GSSG \\
\hline \multirow[t]{12}{*}{ Karrikin pretreatment } & \multirow[t]{3}{*}{0} & 0 & $122.77^{\mathrm{bc}}$ & $35.71^{i}$ & $3.44^{c}$ \\
\hline & & 50 & $122.44^{c}$ & $36.40^{\mathrm{hi}}$ & $3.36^{\mathrm{cd}}$ \\
\hline & & 100 & $122.74^{\mathrm{bc}}$ & $35.99^{i}$ & $3.42^{c}$ \\
\hline & \multirow[t]{3}{*}{50} & 0 & $100.90^{\mathrm{e}}$ & $41.45^{\text {de }}$ & $2.45^{\mathrm{gh}}$ \\
\hline & & 50 & $127.06^{\mathrm{ab}}$ & $30.77^{j}$ & $4.15^{\mathrm{b}}$ \\
\hline & & 100 & $130.56^{\mathrm{a}}$ & $28.11^{\mathrm{j}}$ & $4.67^{\mathrm{a}}$ \\
\hline & \multirow[t]{3}{*}{100} & 0 & $80.62^{h}$ & $50.26^{b c}$ & $1.61^{\mathrm{ij}}$ \\
\hline & & 50 & $99.25^{\text {ef }}$ & $41.89^{\text {de }}$ & $2.38^{\mathrm{gh}}$ \\
\hline & & 100 & $101.73^{\mathrm{e}}$ & $40.42^{\text {defg }}$ & $2.53^{9}$ \\
\hline & \multirow[t]{3}{*}{150} & 0 & $60.62^{j}$ & $55.59^{\mathrm{a}}$ & $1.09^{k}$ \\
\hline & & 50 & $80.38^{h}$ & $50.73^{b} c$ & $1.59^{\mathrm{ij}}$ \\
\hline & & 100 & $79.40^{\mathrm{h}}$ & $47.39^{c}$ & $1.68^{\mathrm{ij}}$ \\
\hline \multirow[t]{12}{*}{ Nonkarrikin pretreatment } & \multirow[t]{3}{*}{0} & 0 & $121.24^{c}$ & $39.68^{\text {efgh }}$ & $3.06^{\text {ef }}$ \\
\hline & & 50 & $122.58^{c}$ & $37.52^{\text {fghi }}$ & $3.27^{\text {cdef }}$ \\
\hline & & 100 & $124.02^{\mathrm{bc}}$ & $37.33^{\mathrm{ghi}}$ & $3.33^{\text {cde }}$ \\
\hline & \multirow[t]{3}{*}{50} & 0 & $95.56^{f}$ & $43.71^{d}$ & $2.19^{h}$ \\
\hline & & 50 & $113.65^{d}$ & $37.76^{\text {fghi }}$ & $3.01^{f}$ \\
\hline & & 100 & $114.59^{d}$ & $37.11^{\mathrm{ghi}}$ & $3.09^{\text {def }}$ \\
\hline & \multirow[t]{3}{*}{100} & 0 & $73.06^{i}$ & $52.08^{b}$ & $1.41^{j}$ \\
\hline & & 50 & $87.09^{9}$ & $48.75^{b c}$ & $1.78^{i}$ \\
\hline & & 100 & $90.44^{\mathrm{g}}$ & $41.05^{\text {def }}$ & $2.21^{\mathrm{h}}$ \\
\hline & \multirow[t]{3}{*}{150} & 0 & $53.41^{\mathrm{k}}$ & $56.82^{\mathrm{a}}$ & $0.94^{k}$ \\
\hline & & 50 & $70.54^{i}$ & $50.14^{b c}$ & $1.40^{\mathrm{j}}$ \\
\hline & & 100 & $70.90^{i}$ & $47.62^{c}$ & $1.49^{j}$ \\
\hline \multicolumn{3}{|l|}{$\operatorname{LSD}_{(0.05)}$} & 4.46 & 3.54 & 0.28 \\
\hline
\end{tabular}

Values (means of three replicates) with different superscripts are significantly different by the LSD multiple range test at $P \leq 0.05$

calcium on oxidative stress in Calligonum mongolicum. Our results also indicated that calcium treatments increased SOD activity under salt stress. This means that calcium boosts SOD activity of plant to protect from superoxide radicals leading to protection against cellular oxidative damage [22]. The application of calcium to salt-stressed black cumin plants also promoted APX activity. Our results agree with those described by other authors who have proposed that calcium application to salt-stressed plants induces increases in antioxidant enzyme activities and GSH content [22, 24]. GSH has an important role in maintaining the GSH/GSSG ratio in the conversion of GSSG to GSH. Karrikin pretreatment of black cumin seeds before salinity treatment and then foliar-applied calcium increased GSH production, thereby converting more GSSG to its reduced form which improved salt stress tolerance of black cumin. These interesting results of presented study were in accordance with the finding of Chan and Lam [58]. Our results indicated that the salt-stressed black cumin cells were negatively affected by accumulating more MDA than those of controls. Foliarapplied calcium to salt-stressed black cumin plants resulted in a reduced content of MDA which was associated with an elevated SOD activity leading to plant protection from oxidative damage. It has been shown that calcium ions relieved cell membrane injury and reduced electrolyte leakage under abiotic stresses [59]. This is in accordance with our results regarding the positive effects of calcium on reducing electrolyte leakage of $\mathrm{NaCl}$-treated black cumin plant cells. The positive correlations between seed and oil yield with GHS as nonenzymatic antioxidant defense system well justify the positive impact of karrikin and $\mathrm{CaCl}_{2}$ on increasing salinity stress resistance by activating antioxidant defense system, heightening the seed and oil production as well as improving oil quality.

\section{Conclusion}

Our results demonstrated the detrimental effects of salinity on seed oil yield and photosynthetic parameters of black cumin plants. It also enhanced the production of ROS and electrolyte leakage. The present findings supported 


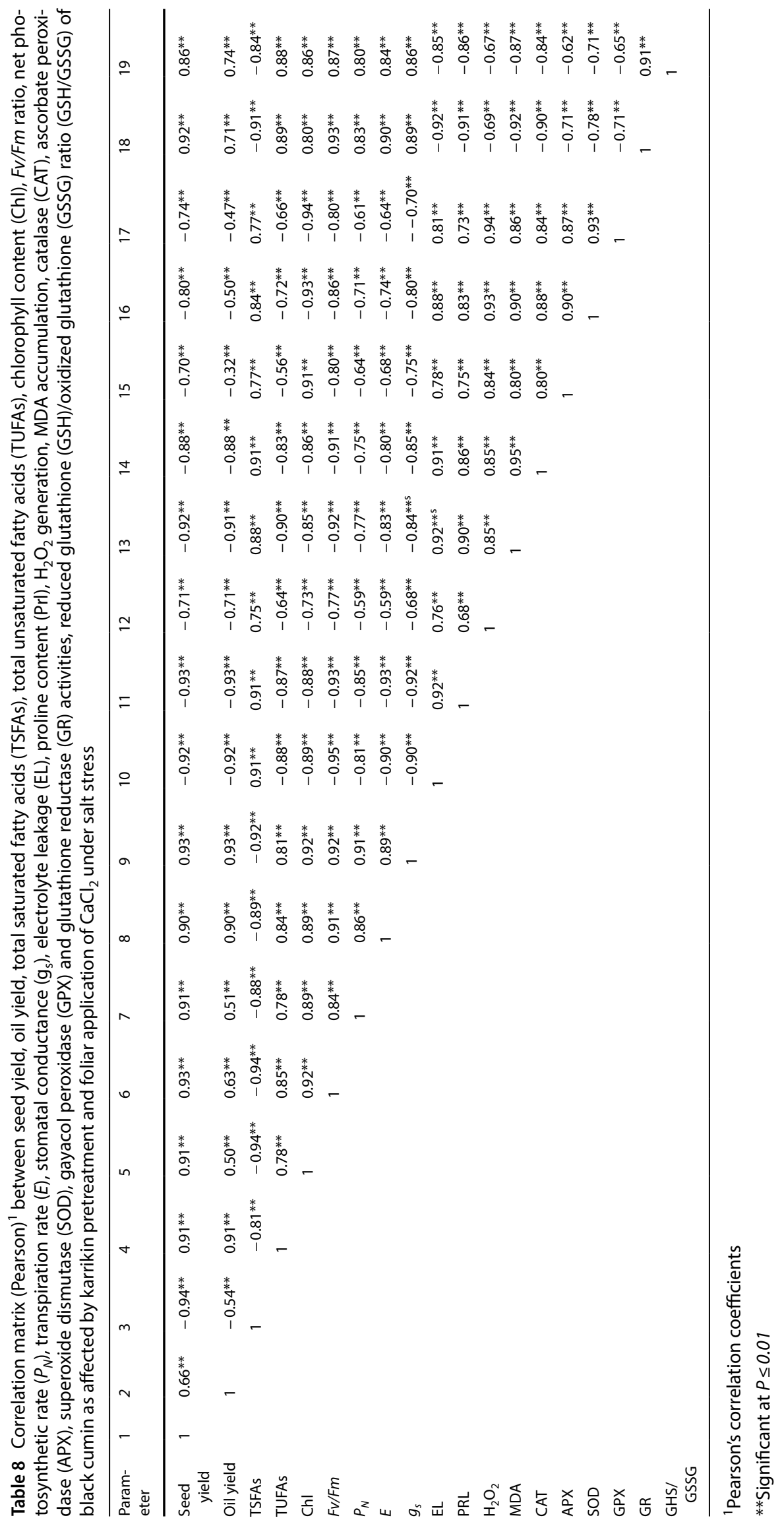


the view that karrikin pretreatment of black cumin seeds followed by calcium foliar application during seedling growth protected Nigella sativa against salinity stress by alleviating the oxidative stress and improved oil yield and composition of seeds. Pretreatment with KARs followed by foliar-applied calcium on $\mathrm{NaCl}$-grown black cumin plants further increased the content of proline, reduced the production of $\mathrm{H}_{2} \mathrm{O}_{2}$ and MDA accumulation and increased the activity of antioxidant enzymes such as CAT, SOD, APX and GR. The results of this experiment showed that pretreatment with KARs followed by calcium foliar application during seedling growth harnessed salt stress and paved the way forward to boost salt-stress tolerance which is vital to future crop productivity.

\section{Compliance with ethical standards}

Conflicts of interest The authors declare that the research was conducted in the absence of any commercial or financial relationships that could be construed as a potential conflict of interest.

\section{References}

1. Datta AK, Saha A, Bhattacharya A, Mandal A, Paul R, Sengupta S (2012) Black cumin (Nigella sativa L.)—a review. J Plant Dev Sci 4(1):1-43

2. Ramadan MF (2007) Nutritional value, functional properties and nutraceutical applications of black cumin (Nigella sativa L.): an overview. Int J Food Sci Technol 42:1208-1218

3. D'Antuono LF, Moretti A, Lovato AFS (2002) Seed yield, yield components, oil content and essential oil content and composition of Nigella sativa L. and Nigella damascena L. Ind Crop Prod 15(1):59-69. https://doi.org/10.1016/S0926-6690(01)00096-6

4. Piras A, Rosa A, Marongiu B, Porcedda S, Falconieri D, Dessi MA, Ozcelik B, Koca U (2013) Chemical composition and in vitro bioactivity of the volatile and fixed oils of Nigella sativa L. extracted by supercritical carbon dioxide. Ind Crop Prod 46:317-323. https ://doi.org/10.1016/j.indcrop.2013.02.013

5. Munns R, Tester M (2008) Mechanisms of salinity tolerance. Annu Rev Plant Biol 59:651-681. https://doi.org/10.1146/annur ev.arplant.59.032607.092911

6. Shabala S, Cuin TA (2008) Potassium transport and plant salt tolerance. Physiol Plant 133:651-669. https://doi.org/10.111 1/j.1399-3054.2007.01008.x

7. Dassanayake M, Larkin JC (2017) Making plants break a sweat: the structure, function, and evolution of plant salt glands. Front Plant Sci 8:406. https://doi.org/10.3389/fpls.2017.00406

8. Ahmad P, Hashem A, Abd-Allah EF, Alqarawi AA, John R, Egamberdieva D, Gucel S (2015) Role of Trichoderma harzianum in mitigating $\mathrm{NaCl}$ stress in Indian mustard (Brassica juncea $\mathrm{L}$ ) through antioxidative defense system. Front Plant Sci 6:868. https://doi. org/10.3389/fpls.2015.00868

9. Fatma M, Masood A, Per TS, Khan NA (2016) Nitric oxide alleviates salt stress inhibited photosynthetic performance by interacting with sulfur assimilation in mustard. Front Plant Sci 7:521. https://doi.org/10.3389/fpls.2016.00521

10. Nair JJ, Munro OQ, Pošta M, Papenfus HB, Beier P, VanStaden $J$ (2012) X-ray crystallographic structure determination of the smoke-derived karrikin KAR3. S Afr J Bot 88:107-109. https:// doi.org/10.1016/j.sajb.2013.06.009

11. Forieri I, Hildebrandt U, Rostás M (2016) Salinity stress effects on direct and indirect defence metabolites in maize. Environ Exp Bot 122:68-77. https://doi.org/10.1016/j.envexpbot.2015.09.007

12. Chiwocha SDS, Dixon KW, Flematti GR, Ghisalberti EL, Merritt DJ, Nelson DC, Riseborough JM, Smith SM, Stevens JC (2009) Karrikins: a new family of plant growth regulators in smoke. Plant Sci 177:252-256

13. Dixon KW, Merritt DJ, Flematti GR, Ghisalberti EL (2009) Karrikinolide-a phytoreactive compound derived from smoke with applications in horticulture, ecological restoration and agriculture. Acta Hortic 813:155-170. https://doi.org/10.17660 /ActaHortic.2009.813.20

14. Halford B (2010) Smoke signals. Chem Eng News 88:37-38

15. Long RL, Stevens JC, Griffiths EM, Adamek M, Powles SB, Merritt DJ (2011) Detecting karrikinolide responses in seeds of the Poaceae. Aust J Bot 59:610-620

16. Wang S, Liu P, Chen D, Yin L, Li H, Deng X (2015) Silicon enhanced salt tolerance by improving the root water uptake and decreasing the ion toxicity in cucumber. Front Plant Sci 6:759. https:// doi.org/10.3389/fpls.2015.00759

17. Kim JM, To TK, Matsui A, Tanoi K, Kobayashi NI, Matsuda F, Habu Y, Ogawa D, Sakamoto T, Matsunaga S, Bashir K, Rasheed S, Ando M, Takeda H, Kawaura K, Kusano M, Fukushima A, Endo TA, Kuromori T, Ishida J, Morosawa T, Tanaka M, Torii C, Takebayashi Y, Sakakibara H, Ogihara Y, Saito K, Shinozaki K, Devoto A, Seki M (2017) Acetate-mediated novel survival strategy against drought in plants. Nat Plants 3:17097

18. Jia KP, Baz L, Al-Babili S (2018) From carotenoids to strigolactones. J Exp Bot 69:2189-2204. https://doi.org/10.1093/jxb/ erx476

19. Banerjee A, Tripathi DK, Roychoudhury A (2019) The karrikin "calisthenics": Can compounds derived from smoke help in stress tolerance? Physiol Plant 165:290-302. https://doi.org/10.1111/ ppl.12836

20. Khan MN, Siddiqui MH, Mohammad F, Naeem M, Khan MMA (2010) Calcium chloride and gibberellic acid protect linseed (Linum usitatissimum L.) from $\mathrm{NaCl}$ stress by inducing antioxidative defence system and osmoprotectant accumulation. Acta Physiol Plant 32:121-132. https://doi.org/10.1007/s1173 8-009-0387-z

21. Hernandez JA, Aguilar AN, Portillo B, Gomez EL, Beneyto JM, Garcia-Legaz MF (2003) The effect of calcium on the antioxidant enzymes from salt-treated loquat and anger plants. Funct Plant Biol 130:1127-1137. https://doi.org/10.1071/FP03098

22. Amor NB, Megdiche W, Jimenez A, Sevilla F, Abdelly C (2010) The effect of calcium on the antioxidant systems in the halophyte Cakile maritima under salt stress. Acta Physiol Plant 32:453-461

23. Wang F, Zeng B, Sun Z, Zhu C (2009) Relationship between proline and $\mathrm{Hg}^{+2}$-induced oxidative stress in tolerant rice mutant. Arch Environ Con Tox 56:723-731. https://doi.org/10.1007/ s00244-008-9226-2

24. Ahmad P, Abd-Allah EF, Alyemeni MN, Wijaya L, Alam P, Bhardwaj $\mathrm{R}$, Siddique KHM (2018) Exogenous application of calcium to 24-epibrassinosteroid pretreated tomato seedlings mitigates $\mathrm{NaCl}$ toxicity by modifying ascorbate-glutathione cycle and secondary metabolites. Sci Rep 8:13515. https://doi.org/10.1038/ s41598-018-31917-1

25. Liu B, Luo C, Li X, Gray L, Zhang F, Liu M (2014) Research on the threshold of aluminum toxicity and the alleviation effects of exogenous calcium, phosphorus, and nitrogen on the growth of Chinese fir seedlings under aluminum stress. Commun Soil Sci Plan 45:126-139

26. Afzal Shah F, Wei X, Wang Q, Liu W, Wang D, Yao Y, Hu H, Chen X, Huang S, Hou J, Lu R, Liu C, Ni J, Wu L (2020) Karrikin improves 
osmotic and salt stress tolerance via the regulation of the redox homeostasis in the oil plant Sapium sebiferum. Front Plant Sci 11:216. https://doi.org/10.3389/fpls.2020.00216

27. Tahjib-UI-Arif M, Roy PR, Sohag AAM, Afrin S, Rady MM, Afzal Hossain M (2019) Exogenous calcium supplementation improves salinity tolerance in BRRI Dhan28; a salt-susceptible high-yielding Oryza Sativa Cultivar. J Crop Sci Biotechnol 21:383-394

28. Khalid KA (2017) Changes in lipids and fatty acids of Nigella sativa L. under salinity stress. J Mater Environ Sci 8(10):3502-3507

29. Arnon DI (1949) Copper enzymes in isolated chloroplasts phenoloxidase in Beta vulgaris. Plant Physiol 24:1

30. Ozden M, Demirel U, Kahraman A (2009) Effects of proline on antioxidant system in leaves of grapevine (Vitis vinifera $\mathrm{L}$.) exposed to oxidative stress by $\mathrm{H}_{2} \mathrm{O}_{2}$. Sci Hortic 119:163-168. https://doi.org/10.1016/j.scienta.2008.07.031

31. Velikova V, Loreto F (2005) On the relationship between isoprene emission and thermo tolerance in Phragmites ausrralis leaves exposed to high temperatures and during the recovery from a heat stress. Plant Cell Environ 28(3):318-327. https:// doi.org/10.1111/j.1365-3040.2004.01314.x

32. Bates LS, Waldren EP, Teare ID (1973) Rapid determination of free proline for water stress studies. Plant Soil 39:205-207. https://doi.org/10.1007/BF00018060

33. Hasanuzzaman M, Hossain MA, Fujita M (2011) Nitric oxide modulates antioxidant defense and the methylglyoxal detoxification system and reduces salinity-induced damage of wheat seedlings. Plant Biotecnol Rep 5:353-365. https://doi. org/10.1007/s11816-011-0189-9

34. Cavalcanti FR, Oliveira JT, Martins-miranda AAS, Viegas RA, Silveira JAG (2004) Superoxide dismutase, catalase and peroxidase activities do not confer protection against oxidative damage in salt-stressed cowpea leaves. New Phytol 163:563571. https://doi.org/10.1111/j.1469-8137.2004.01139.x

35. Nakano Y, Asada K (1981) Hydrogen peroxide is scavenged by ascorbate-specific peroxidase in spinach chloroplasts. Plant Cell Physiol 22:867-880

36. Lopez-Vargas ER, Ortega-Ortiz H, Cadenas-Pliego G, de Alba RK, de la Fuente MC, Benavides-Mendoza A, Juarez-Maldonado A (2018) Foliar application of copper nanoparticles increases the fruit quality and the content of bioactive compounds in tomatoes. Appl Sci 8:1020. https://doi.org/10.3390/ app8071020

37. Goli SAH, Sahafi SM, Rahimmalek RB, M, (2013) Novel oilseed of Dracocephalum kotschyi with high n-3 to n-6 polyunsaturated fatty acid ratio. Ind Crops Prod 43:188-193. https://doi. org/10.1016/j.indcrop.2012.07.036

38. Rebey IB, Bourgou S, Rahali FZ, Msaada K, Ksouri R, Marzouk B (2017) Relation between salt tolerance and biochemical changes in cumin (Cuminum cyminum L.) seeds. J Food Drug Anal 25:391-402

39. Rahimi R, Mohammolakhani A, Roohi V, Armand N (2012) Effects of salt stress on the yield components, essential oil content and cholorophyll concentration of three fennel populations. Intl J Agron Plant Prod 3:716-720

40. Ashraf M, Orooj A (2006) Salt stress effects on growth, ion accumulation and seed oil concentration in an arid zone traditional medicinal plant ajwain (Trachyspermum ammoli [L.] Sprague). J Arid Environ 64:209-220

41. Zhu JK (2001) Plant salt tolerance. Trends in Plant Sci 6:66-71. https://doi.org/10.1016/s1360-1385(00)01838-0

42. Rahman A, Nahar K, Hasanuzzaman M, Fujita M (2016) Calcium supplementation improves $\mathrm{Na}^{+} / \mathrm{K}^{+}$ratio, antioxidant defense and glyoxalase systems in salt-stressed rice seedlings. Front Plant Sci 7:609. https://doi.org/10.3389/fpls.2016.00609
43. Hojjatnooghi F, Mozafari V, Tajabadipour A, Hokmabadi H (2014) Effects of salinity and calcium on the growth and chemical composition of pistachio seedlings. J Plant Nutr 37:928-941. https:// doi.org/10.1080/01904167.2014.888737

44. Gigon A, Matos AR, Laffray D, Zuily-Fodil Y, Pham-Thi AT (2004) Effect of drought stress on lipid metabolism in the leaves of Arabidopsis thaliana (ecotype Columbia). Ann Bot 94:345-351. https://doi.org/10.1093/aob/mch150

45. Malkit A, Sadka A, Fisher M, Goldshlag P, Gokhman I, Zamir A (2002) Salt induction of fatty acid elongase and membrane lipid modifications in the extreme halotolerant alga Dunaliella salina. Plant Physiol 129:1320-1329. https://doi.org/10.1104/pp.00190 9

46. Xu D, Wang W, Gao T, Fang X, Gao X, Li J, Bu H, Mu J (2017) Calcium alleviates decreases in photosynthesis under salt stress by enhancing antioxidant metabolism and adjusting solute accumulation in Calligonum mongolicum. Conserv Physiol 5(1):1-8. https://doi.org/10.1093/conphys/cox060

47. Parveen N, Ashraf M (2010) Role of silicon in mitigating the adverse effects of salt stress on growth and photosynthetic attributes of two maize (Zea mays L.) cultivars grown hydroponically. Pak J Bot 42:1675-1684

48. Xu C, Li X, Zhang L (2013) The effect of calcium chloride on growth, photosynthesis, and antioxidant responses of Zoysia japonica under drought conditions. PLoS ONE 8:e68214. https ://doi.org/10.1371/journal.pone.0068214

49. Per TS, Khan NA, Reddy PS, Masood A, Hasanuzzaman M, Khan MIR, Anjum NA (2017) Approaches in modulating proline metabolism in plants for salt and drought stress tolerance: Phytohormones, mineral nutrients and transgenics. Plant Physiol Biochem 115:126-140. https://doi.org/10.1016/j.plaph y.2017.03.018

50. Ghebrehiwot HM, Kulkarni MG, Kirkman KP, Van Staden J (2008) Smoke-water and a smoke-isolated butenolide improve germination and seedling vigour of Eragrostis tef (Zucc.) trotter under high temperature and low osmotic potential. J Agron Crop Sci 194:270-277. https://doi.org/10.1111/j.1439-037X.2008.00321 .$x$

51. Jamil M, Kanwal M, Aslam MM, Khan SU, Malook I, Tu J, Rehman SU (2014) Effect of plant-derived smoke priming on physiological and biochemical characteristics of rice under salt stress condition. Aust J Crop Sci 8:159-170

52. Smith SM, Li J (2014) Signalling and responses to strigolactones and karrikins. Curr Opin Plant Biol 21:23-29. https://doi. org/10.1016/j.pbi.2014.06.003

53. Ren CG, Kong CC, Xie ZH (2018) Role of abscisic acid in strigolactone-induced salt stress tolerance in arbuscular mycorrhizal Sesbania cannabina seedlings. BMC Plant Biol 18(1):47. https:// doi.org/10.1186/s12870-018-1292-7

54. Ma N, Hu C, Wan L, Hu Q, Xiong JL, Zhang CL (2017) Strigolactones improve plant growth, photosynthesis, and alleviate oxidative stress under salinity in rapeseed (Brassica napus L.) by regulating gene expression. Front Plant Sci 8:1671. https:// doi.org/10.3389/fpls.2017.01671

55. Mostofa MG, Li W, Nguyen KH, Fujita M, Tran LP (2018) Strigolactones in plant adaptation to abiotic stresses: an emerging avenue of plant research. Plant Cell Environ 41:2227-2243. https ://doi.org/10.1111/pce.13364

56. Sunmonu TO, Kulkarni MG, VanStaden J (2016) Smoke-water, karrikinolide and gibberellic acid stimulate growth in bean and maize seedlings by efficient starch mobilization and suppression of oxidative stress. S Afr J Bot 102:4-11. https://doi. org/10.1016/j.sajb.2015.06.015

57. Ha CV, Leyva Gonzalez MA, Osakabe Y, Tran UT, Nishiyama R, Watanabe Y, Tanaka M, Seki M, Yamaguchi S, Dong NV, Yamaguchi Shinozaki K, Shinozaki K, Herrera Estrella L, Tran LS (2014) 
Positive regulatory role of strigolactone in plant responses to drought and salt stress. Proc Nat Acad Sci USA 111(2):851-856

58. Chan C (2014) Lam HM (2014) A putative lambda class glutathione s-transferase enhances plant survival under salinity stress. Plant Cell Physiol 55:570-579. https://doi.org/10.1093/ pcp/pct201

59. Guimaraes FVA, de Lacerda CF, Marques EC, de Miranda MRA, de Abreu CEB, Prisco JT, Gomes-Filho E (2011) Calcium can moderate changes on membrane structure and lipid composition in cowpea plants under salt stress. Plant Growth Regul 65:55-63
Publisher's Note Springer Nature remains neutral with regard to jurisdictional claims in published maps and institutional affiliations.

\section{SN Applied Sciences}

